\title{
Simultaneous Transfer of Energy and Information for MIMO-OFDM Relay System
}

\author{
Batu K. Chalise, Yimin D. Zhang and Moeness G. Amin
}

\begin{abstract}
This paper investigates performance tradeoffs between energy and information transfer in a relay based twohop wireless system involving an energy harvesting receiver. The source and relay nodes of the two-hop amplify-and-forward (AF) system employ orthogonal space-time block codes along with orthogonal frequency-division multiplexing (OFDM) scheme. The joint optimal source and relay precoders over the subchannels in frequencies are designed to achieve different tradeoffs between the overall energy transfer capability and the information rate, which are characterized by the boundary of the so-called rateenergy (R-E) region. The effect of various parameters on the boundary of the $R-E$ region is demonstrated for different frequency selective channel models.
\end{abstract}

Index Terms-MIMO-OFDM, energy harvesting, information and power transfer, OSTBC, and AF relay.

\section{INTRODUCTION}

Recently, the technique of harvesting energy from radio signals has received much attention in various applications, such as radio-frequency identification systems [1] and body sensor networks with medical implants [2]. Energy harvesting (EH) can be used for prolonging the network lifetime operation of energy-constrained sensor networks which typically employ small batteries having limited power-supply capability and duration. This has motivated the authors of [3] to propose a wireless communication system in which some nodes do not possess reliable power supplies, and therefore, have to harvest energy from signals transmitted by other nodes. In particular, [3] considers a three-node multiple-input multipleoutput (MIMO) system in which one receiver node decodes information whereas the other node harvests energy using the signals broadcast by a common transmitting node. The transmitter attempts to simultaneously maximize the information transfer to the intended receiver and the power transfer to the EH receiver. Note that [3] extends the study of simultaneous information and power transfer of [4], [5] from the singleinput single-output (SISO) link in the co-located receiver (i.e., information and $\mathrm{EH}$ receivers are the same) case to the multiantenna setup with both co-located and separated receivers.

In this paper, different from previous contributions [3], [4], [5], we consider a wireless communication system in which a multi-antenna EH receiver exists in the vicinity of a two-hop MIMO relay system where both the source and relay nodes employ orthogonal space-time block codes (OSTBCs) [7] and precoders for data transmission. In contrast to the assumption of flat-fading channels [6], we consider frequency selectivity,

The authors are with the Center for Advanced Communications, Villanova University, Villanova, PA 19085, USA, emails: \{batu.chalise, yimin.zhang, moeness.amin\}@villanova.edu which is most representative for radio channels subject to multipath fading. To this end, we employ orthogonal frequencydivision multiplexing (OFDM) and transmit information over different frequency bands or subcarriers. Further, the fact that OSTBCs significantly simplify optimal decoding without incurring rate-loss for specific case, such as the case with the Alamouti code [7], has motivated us to employ OSTBCs on top of the source and relay precoders for each subcarrier. The relay/destination node of the two-hop system uses maximumratio combining (MRC) technique for detecting/decoding the source signal. In overall, the relay operates in a half-duplex mode using an amplify-and-forward (AF) protocol. The EH receiver harvests energy from the radio signals transmitted by both the source and relay. Information transfer to the destination node and power transfer to the $\mathrm{EH}$ receiver are optimally controlled by properly designing the source and relay precoders in a subcarrier basis. In particular, under the total power constraint of the source and relay, we design both precoders to maximize the rate for the intended receiver while keeping the power transfer to the EH receiver above a certain predefined value. This value is varied to obtain the boundary of the rate-energy (R-E) region which illustrates the tradeoffs between information and energy transfer capability of the relay system. Although power allocation schemes for single user MIMO-OFDM relay system [8] and MIMO relay precoder designs for flat-fading multiuser systems [9] have been considered, to the best our knowledge, simultaneous transfer of energy and information has not been addressed before for frequency selective channels.

The remaining of this paper is organized as follows. In Section II, the proposed system model is described, whereas in Section III, the solutions for the precoder design optimization problems are provided for different transmission strategies. Numerical simulation results are given in Section IV and concluding remarks are made in Section V.

Notations: Upper (lower) bold face letters are used for matrices (vectors); $(\cdot)^{H}, \mathrm{E}\{\cdot\}$, and $\|\cdot\|$ denote Hermitian transpose, mathematical expectation and Frobenius norm, respectively. $\operatorname{tr}(\cdot), \mathcal{C}^{M \times M}$, and $\mathbf{A} \succeq 0$ denote the matrix trace operator, space of $M \times M$ matrices with complex entries, and positive semidefiniteness of $\mathbf{A}$, respectively.

\section{SySTEM MOdeL}

We consider a system shown in Fig. 1, which consists of a multi-antenna two-hop relaying system with a source, a relay, and a destination which is also referred to as an information decoding (ID) receiver, and an EH receiver. The direct 
link between the source and ID receiver is not considered, because we assume that the effects of path attenuation and shadowing are more severe on the direct link when compared to the link via relay. Since, the relay operates in a halfduplex mode, signal transmissions over source-relay (S-R) and relay-ID receiver (R-ID) channels take place in two phases. It is assumed that the available bandwidth is divided into $N$ subcarriers so that each subchannel is basically a flatfading channel, i.e., the separation between two subcarriers (subchannel) is smaller than the coherence bandwidth of the channel. Moreover, the OFDM guard interval is assumed to be larger than the maximum path delay of the channels. Under these assumptions, the OFDM transmission can be analyzed in a subcarrier basis. In the first phase of transmission, the source encodes the input signal of the $n$th subcarrier $(n \in \mathcal{N}=$ $\{1, \cdots, N\})$ using an OSTBC, precodes the encoded signal and transmits the resulting signal to the relay. The $n_{\mathrm{r}} \times T$ matrix of received signal samples at the relay for the $n$th subcarrier can be given by

$$
\mathbf{Y}_{\mathrm{r}, n}=\mathbf{H}_{1, n, \mathrm{I}} \mathbf{F}_{1, n} \mathbf{C}\left(\mathbf{s}_{n}\right)+\mathbf{V}_{\mathrm{r}, n}
$$

where $\mathbf{s}_{n}=\left[s_{1, n} \cdots, s_{K, n}\right]^{T}$ is $K \times 1$ information-bearing complex symbol vector, $\mathbf{C}\left(\mathbf{s}_{n}\right)$ is the $n_{\mathrm{s}} \times T$ OSTBC matrix formed from $\mathbf{s}_{n}, T$ is the number of time periods used for transmitting $\mathbf{s}_{n}, \mathbf{H}_{1, n, \mathrm{I}} \in \mathcal{C}^{n_{\mathrm{r}} \times n_{\mathrm{s}}}$ is the S-R MIMO channel (in frequency domain), $\mathbf{F}_{1, n} \in \mathcal{C}^{n_{\mathrm{s}} \times n_{\mathrm{s}}}$ is the source precoder, and $\mathbf{V}_{\mathrm{r}, n} \in \mathcal{C}^{n_{\mathrm{r}} \times T}$ is the matrix of zero-mean circularly symmetric complex Gaussian (ZMCSCG) elements with variance $\widetilde{\sigma}_{1, n}^{2}$. It is assumed that $\left\{s_{k, n}\right\}_{k=1}^{K}$ are chosen from signal constellations with $\mathrm{E}\left\{\left|s_{k, n}\right|^{2}\right\}=1$. Due to the orthogonality of the OSTBC, $\mathbf{C}\left(\mathbf{s}_{n}\right)$ fulfills the property $\mathbf{C}\left(\mathbf{s}_{n}\right) \mathbf{C}^{H}\left(\mathbf{s}_{n}\right)=a\left\|\mathbf{s}_{n}\right\|^{2} \mathbf{I}_{n_{\mathrm{s}}}$, where the constant $a$ (e.g, $a=1$ for the Alamouti code [7]) depends on the chosen OSTBC matrix. The transmit power of the source and the energy received by the $\mathrm{EH}$ receiver on the $n$th subcarrier during the first phase of the two-hop transmission can be, respectively, given by

$$
\begin{aligned}
P_{\mathrm{s}, n} & =a K \operatorname{tr}\left(\mathbf{F}_{1, n} \mathbf{F}_{1, n}^{H}\right), \\
P_{\mathrm{e}, 1, n} & =a K \operatorname{tr}\left(\mathbf{H}_{1, n, \mathrm{E}} \mathbf{F}_{1, n} \mathbf{F}_{1, n}^{H} \mathbf{H}_{1, n, \mathrm{E}}^{H}\right)
\end{aligned}
$$

where $\mathbf{H}_{1, n, \mathrm{E}} \in \mathcal{C}^{n_{\mathrm{e}} \times n_{\mathrm{s}}}$ is the MIMO channel between the source and EH receiver corresponding to $n$th subcarrier. Due to the application of the OSTBC at the source and the MRC scheme at the relay during the first phase of signal transmission, the S-R MIMO channel of $n$th subcarrier is decoupled into $K$ parallel SISO channels. Thus, the signal received by the relay on the $k$ th S-R SISO channel corresponding to $n$th subcarrier is given by [10]

$$
y_{k, n}^{\mathrm{R}}=\left\|\mathbf{H}_{1, n, \mathrm{I}} \mathbf{F}_{1, n}\right\| s_{k, n}+v_{1, k, n}, k \in\{1, \cdots, K\}
$$

where $v_{1, k, n} \sim \mathcal{N}_{C}\left(0, \sigma_{1, n}^{2}\right)$ is the additive Gaussian noise at the relay for the $k$ th S-R SISO channel and $\sigma_{1, n}^{2}=\widetilde{\sigma}_{1, n}^{2} / a$. The relay normalizes $\left\{y_{k, n}^{\mathrm{R}}\right\}_{k=1}^{K}$ yielding

$$
\widetilde{y}_{k, n}^{\mathrm{R}}=\frac{y_{k, n}^{\mathrm{R}}}{\sqrt{\mathrm{E}\left\{\left|y_{k, n}^{\mathrm{R}}\right|^{2}\right\}}}=\frac{\left\|\mathbf{H}_{1, n, \mathrm{I}} \mathbf{F}_{1, n}\right\| s_{k, n}+v_{1, k, n}}{\sqrt{\left\|\mathbf{H}_{1, n, \mathrm{I}} \mathbf{F}_{1, n}\right\|^{2}+\sigma_{1, n}^{2}}} .
$$

The relay then employs OSTBC to encode $\left\{\widetilde{y}_{k, n}^{\mathrm{R}}\right\}_{k=1}^{K}$ and precodes the resulting OSTBC encoded signal. The output of the relay for the $n$th subcarrier is thus given by $\mathbf{Y}_{\mathrm{ro}, n}=$ $\mathbf{F}_{2, n} \mathbf{C}\left(\widetilde{\mathbf{y}}_{n}\right)$ where $\mathbf{F}_{2, n} \in \mathcal{C}^{n_{\mathrm{r}} \times n_{\mathrm{r}}}$ is the relay precoder, $\widetilde{\mathbf{y}}_{n}=$ $\left[\widetilde{y}_{1, n}^{\mathrm{R}}, \cdots, \widetilde{y}_{K, n}^{\mathrm{R}}\right]^{T}, \mathbf{C}\left(\widetilde{\mathbf{y}}_{n}\right) \in \mathcal{C}^{n_{\mathrm{r}} \times T}$ is the OSTBC obtained after encoding $\widetilde{\mathbf{y}}_{n}$ and satisfies the relation $\mathbf{C}\left(\widetilde{\mathbf{y}}_{n}\right) \mathbf{C}^{H}\left(\widetilde{\mathbf{y}}_{n}\right)=$ $a|| \widetilde{\mathbf{y}}_{n} \|^{2} \mathbf{I}_{n_{\mathrm{r}}}$. The transmit power of the relay and the energy received by the EH receiver on the $n$th subcarrier during the second phase can be thus, respectively, given by

$$
\begin{aligned}
& P_{\mathrm{r}, n}=a K \operatorname{tr}\left(\mathbf{F}_{2, n} \mathbf{F}_{2, n}^{H}\right), \\
& P_{\mathrm{e}, 2, n}=a K \operatorname{tr}\left(\mathbf{H}_{2, n, \mathrm{E}} \mathbf{F}_{2, n} \mathbf{F}_{2, n}^{H} \mathbf{H}_{2, n, \mathrm{E}}^{H}\right) .
\end{aligned}
$$

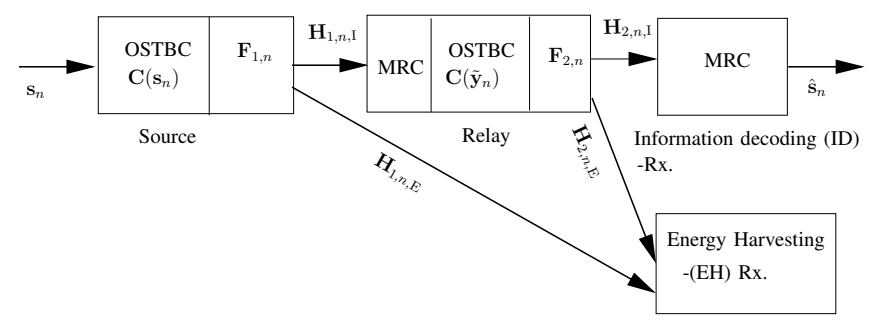

Fig. 1. Two-hop OSTBC based relay system with EH Rx.

The $n_{\mathrm{d}} \times T$ matrix of signal samples received at the ID receiver on the $n$th subcarrier during the second phase of transmission can be written as $\mathbf{Y}_{\mathrm{d}, n}=\mathbf{H}_{2, n, \mathrm{I}} \mathbf{F}_{2, n} \mathbf{C}\left(\widetilde{\mathbf{y}}_{n}\right)+$ $\mathbf{V}_{\mathrm{d}, n}$, where $\mathbf{H}_{2, n, \mathrm{I}} \in \mathcal{C}^{n_{\mathrm{d}} \times n_{\mathrm{r}}}$ is the R-ID MIMO channel and $\mathbf{V}_{\mathrm{d}, n} \in \mathcal{C}^{n_{\mathrm{d}} \times T}$ is the matrix of ZMCSCG elements with variance $\widetilde{\sigma}_{2, n}^{2}$. The ID receiver uses MRC to detect the source signals. Due to the application of the OSTBC at the relay and MRC at the ID receiver, the R-ID MIMO channel also turns into $K$ parallel SISO channels. Thus, the signal received by the ID receiver on the $k$ th R-ID SISO channel corresponding to the $n$th subcarrier can be expressed as

$$
y_{k, n}^{\mathrm{D}}=\left\|\mathbf{H}_{2, n, \mathrm{I}} \mathbf{F}_{2, n}\right\| \widetilde{y}_{k, n}^{\mathrm{R}}+v_{2, k, n}
$$

where $v_{2, k, n} \sim \mathcal{N}_{C}\left(0, \sigma_{2, n}^{2}\right)$ is the additive Gaussian noise at the ID receiver for the $k$ th channel and $\sigma_{2, n}^{2}=\widetilde{\sigma}_{2, n}^{2} / a$. With the help of (4), (6) can be written as

$$
\begin{aligned}
y_{k, n}^{\mathrm{D}}= & \frac{\left\|\mathbf{H}_{2, n, \mathrm{I}} \mathbf{F}_{2, n}\right\|\left\|\mathbf{H}_{1, n, \mathrm{I}} \mathbf{F}_{1, n}\right\| s_{k, n}+\left\|\mathbf{H}_{2, n, \mathrm{I}} \mathbf{F}_{2, n}\right\| v_{1, k, n}}{\sqrt{\left\|\mathbf{H}_{1, n, \mathrm{I}} \mathbf{F}_{1, n}\right\|^{2}+\sigma_{1, n}^{2}}} \\
& +v_{2, k, n} .
\end{aligned}
$$

The signal-to-noise ratio (SNR) at the ID receiver for the $n$th subcarrier can be expressed as

$$
\begin{aligned}
\gamma_{n} & =\frac{\left\|\mathbf{H}_{2, n, \mathrm{I}} \mathbf{F}_{2, n}\right\|^{2}\left\|\mathbf{H}_{1, n, \mathrm{I}} \mathbf{F}_{1, n}\right\|^{2}}{\left\|\mathbf{H}_{2, n, \mathrm{I}} \mathbf{F}_{2, n}\right\|^{2} \sigma_{1, n}^{2}+\left\|\mathbf{H}_{1, n, \mathrm{I}} \mathbf{F}_{1, n}\right\|^{2} \sigma_{2, n}^{2}+\sigma_{1, n}^{2} \sigma_{2, n}^{2}} \\
& =\frac{\gamma_{1, n} \gamma_{2, n}}{\gamma_{1, n}+\gamma_{2, n}+1}
\end{aligned}
$$

where $\gamma_{i, n}=\frac{\left\|\mathbf{H}_{i, n, \mathrm{I}} \mathbf{F}_{i, n}\right\|^{2}}{\sigma_{i, n}^{2}}$ for $i=1,2$ and $n \in \mathcal{N}$. 


\section{Proposed Transmission Strategies}

In this section, we determine joint optimal precoders $\mathbf{F}_{1, n}$ and $\mathbf{F}_{2, n}$ over all subcarriers for separately maximizing energy transfer to the $\mathrm{EH}$ receiver and information transfer to the ID receiver. We then consider the problem of optimizing the precoders when both receivers are present.

\section{A. Optimization with only $\mathrm{EH}$ receiver}

Consider the MIMO links from the source and relay to the $\mathrm{EH}$ receiver, when there is no ID receiver. In this case, the goal is to design $\mathbf{F}_{1, n}$ and $\mathbf{F}_{2, n}$ for maximizing the total power $\sum_{n=1}^{N} P_{\mathrm{e}, 1, n}+P_{\mathrm{e}, 2, n}$ received at the $\mathrm{EH}$ receiver. This design problem can be formulated as

$$
\begin{aligned}
& \mathcal{P}_{1}: \max _{\left\{\mathbf{F}_{1, n}, \mathbf{F}_{2, n}, n \in \mathcal{N}\right\}} \sum_{n=1}^{N}\left\{\operatorname{tr}\left(\mathbf{H}_{1, n, \mathrm{E}} \mathbf{F}_{1, n} \mathbf{F}_{1, n}^{H} \mathbf{H}_{1, n, \mathrm{E}}^{H}\right)+\right. \\
& \left.\operatorname{tr}\left(\mathbf{H}_{2, n, \mathrm{E}} \mathbf{F}_{2, n} \mathbf{F}_{2, n}^{H} \mathbf{H}_{2, n, \mathrm{E}}^{H}\right)\right\} \\
& \text { s.t. } \sum_{n=1}^{N} \operatorname{tr}\left(\mathbf{F}_{1, n} \mathbf{F}_{1, n}^{H}\right)+\operatorname{tr}\left(\mathbf{F}_{2, n} \mathbf{F}_{2, n}^{H}\right) \leq P_{\mathrm{T}}
\end{aligned}
$$

where the constant $a K$ is omitted from the objective function, and $P_{\mathrm{T}}$ is given by $P_{\mathrm{T}}=\frac{\widetilde{P}_{\mathrm{T}}}{a K}$, where $\widetilde{P}_{\mathrm{T}}$ is the total power (source and relay). Let the eigen-decomposition (ED) of $\mathbf{H}_{i, n, \mathrm{E}}^{H} \mathbf{H}_{i, n, \mathrm{E}}$ be given by $\mathbf{H}_{i, n, \mathrm{E}}^{H} \mathbf{H}_{i, n, \mathrm{E}}=\mathbf{U}_{\mathbf{H}_{i, n, \mathrm{E}}} \boldsymbol{\Lambda}_{\mathbf{H}_{i, n, \mathrm{E}}} \mathbf{U}_{\mathbf{H}_{i, n, \mathrm{E}}}^{H}$ with eigenvalues $\lambda_{k}^{\mathbf{H}_{i, n, \mathrm{E}}}\left(k=1, \cdot \cdot, r_{i} \triangleq \operatorname{rank}\left(\mathbf{H}_{i, n, \mathrm{E}}\right)\right)$, in the non-decreasing order, where $i=1,2$. Let $\left\{\mathbf{u}_{i, n, \mathrm{E}}\right\}_{i=1}^{2}$ be the column vectors of $\left\{\mathbf{U}_{\mathbf{H}_{i, n, \mathrm{E}}}\right\}_{i=1}^{2}$ corresponding to $\left\{\lambda_{1}^{\mathbf{H}_{i, n, \mathrm{E}}}\right\}_{i=1}^{2}$.

Proposition 1: The optimal solutions to $\mathcal{P}_{1}$ are either

$$
\begin{aligned}
\widehat{\mathbf{F}}_{1, \widehat{n}}= & {\left[\sqrt{P_{\mathrm{T}}} \mathbf{u}_{1, \widehat{n}, \mathrm{E}}, \mathbf{0}, \cdots, \mathbf{0}\right],\left[\widehat{\mathbf{F}}_{1, \widehat{n}}\right]_{\widetilde{n} \neq \widehat{n}, \widetilde{n}=1}^{N}=\mathbf{0}, } \\
& {\left[\widehat{\mathbf{F}}_{2, n}\right]_{n=1}^{N}=\mathbf{0}, \text { if } \lambda_{1}^{\mathbf{H}_{1, \widehat{n}, \mathrm{E}}} \geq \lambda_{1}^{\mathbf{H}_{1, \widetilde{n}, \mathrm{E}}} \geq \max _{n} \lambda_{1}^{\mathbf{H}_{2, n, \mathrm{E}},}, }
\end{aligned}
$$

$$
\begin{aligned}
\widehat{\mathbf{F}}_{2, \widehat{n}}= & {\left[\sqrt{P_{\mathrm{T}}} \mathbf{u}_{2, \widehat{n}, \mathrm{E}}, \mathbf{0}, \cdots, \mathbf{0}\right],\left[\widehat{\mathbf{F}}_{2, \widetilde{n}}\right]_{\widetilde{n} \neq \widehat{n}, \widetilde{n}=1}^{N}=\mathbf{0}, } \\
& {\left[\widehat{\mathbf{F}}_{1, n}\right]_{n=1}^{N}=\mathbf{0}, \text { if } \lambda_{1}^{\mathbf{H}_{2, \widehat{n}, \mathrm{E}}} \geq \lambda_{1}^{\mathbf{H}_{2, \widetilde{n}, \mathrm{E}}} \geq \max _{n} \lambda_{1}^{\mathbf{H}_{1, n, \mathrm{E}}} . }
\end{aligned}
$$

Proof: Please refer to Appendix A.

It is clear from Proposition 1 that the total power is allocated to the subcarrier corresponding to the best channel from all S-EH and R-EH channels.

\section{B. Optimization with only ID receiver}

Consider the two-hop MIMO relay link from the source to the ID receiver, when no EH receiver exists. The optimal $\mathbf{F}_{1, n}$ and $\mathbf{F}_{2, n}$ that maximize the information rate over the two-hop
MIMO channel is obtained by solving the following problem

$$
\begin{aligned}
& \mathcal{P}_{2}: R_{\max } \triangleq \max _{\left\{\mathbf{F}_{1, n}, \mathbf{F}_{2, n}, \gamma_{1, n}, \gamma_{2, n}, n \in \mathcal{N}\right\}} \frac{R_{\mathrm{c}}}{2 N} \sum_{n=1}^{N} \ln _{2}\{1+ \\
& \left.\frac{\gamma_{1, n} \gamma_{2, n}}{\gamma_{1, n}+\gamma_{2, n}+1}\right\} \\
& \text { s.t } \gamma_{1, n}=\frac{\left\|\mathbf{H}_{1, n, \mathrm{I}} \mathbf{F}_{1, n}\right\|^{2}}{\sigma_{1, n}^{2}}, \gamma_{2, n}=\frac{\left\|\mathbf{H}_{2, n, \mathrm{I}} \mathbf{F}_{2, n}\right\|^{2}}{\sigma_{2, n}^{2}}, \forall n,(12) \\
& \sum_{n=1}^{N} \operatorname{tr}\left(\mathbf{F}_{1, n} \mathbf{F}_{1, n}^{H}\right)+\operatorname{tr}\left(\mathbf{F}_{2, n} \mathbf{F}_{2, n}^{H}\right) \leq P_{\mathrm{T}},
\end{aligned}
$$

where $R_{\mathrm{c}}$ is the code rate of the OSTBC (i.e., $R_{\mathrm{c}}=\frac{K}{T}$ ) and the factor $\frac{1}{2}$ is due to the half-duplex relay. Let the ED of $\mathbf{H}_{j, n, \mathrm{I}}^{H} \mathbf{H}_{j, n, \mathrm{I}}$ be given by $\mathbf{H}_{j, n, \mathrm{I}}^{H} \mathbf{H}_{j, n, \mathrm{I}}=\mathbf{U}_{\mathbf{H}_{j, n, \mathrm{I}}} \boldsymbol{\Lambda}_{\mathbf{H}_{j, n, \mathrm{I}}} \mathbf{U}_{\mathbf{H}_{j, n, \mathrm{I}}}^{H}$ with the eigenvalues $\lambda_{k}^{\mathbf{H}_{j, n, \mathrm{I}}}\left(k=1, \cdot \cdot r_{j} \triangleq \operatorname{rank}\left(\mathbf{H}_{j, n, \mathrm{I}}\right)\right)$ in the non-decreasing order, where $j=1,2$. Let $\left\{\mathbf{u}_{j, n, I}\right\}_{j=1}^{2}$ be the column vectors of $\left\{\mathbf{U}_{\mathbf{H}_{j, n, \mathrm{I}}}\right\}_{j=1}^{2}$ corresponding to $\left\{\lambda_{1}^{\mathbf{H}_{j, n, \mathrm{I}}}\right\}_{j=1}^{2}$. It can be proved (the proof is omitted for brevity) that the optimal $\mathbf{F}_{1, n}$ and $\mathbf{F}_{2, n}$ in $\mathcal{P}_{2}$ are

$$
\begin{aligned}
\overline{\mathbf{F}}_{1, n} & =\left[\sqrt{a_{1, n}} \mathbf{u}_{1, n, \mathrm{I}}, \mathbf{0}, \cdots, \mathbf{0}\right], \\
\overline{\mathbf{F}}_{2, n} & =\left[\sqrt{a_{2, n}} \mathbf{u}_{2, n, \mathrm{I}}, \mathbf{0}, \cdots, \mathbf{0}\right], \forall n \in \mathcal{N}
\end{aligned}
$$

where $a_{1, n} \geq 0, a_{2, n} \geq 0, \forall n$ need to be optimized for $\mathcal{P}_{2}$. This leads to the following optimization problem for $\mathcal{P}_{2}$ :

$$
\begin{aligned}
& \overline{\mathcal{P}}_{2}: R_{\max } \triangleq \max _{\left\{a_{1, n}, a_{2, n}, n \in \mathcal{N}\right\}} \frac{R_{\mathrm{c}}}{2 N} \sum_{n=1}^{N} \ln 2\{1+ \\
& \left.\frac{a_{1, n} a_{2, n} \widetilde{\lambda}_{1}^{\mathbf{H}_{1, n, \mathrm{I}}} \widetilde{\lambda}_{1}^{\mathbf{H}_{2, n, \mathrm{I}}}}{a_{1, n} \widetilde{\lambda}_{1}^{\mathbf{H}_{1, n, \mathrm{I}}}+a_{2, n} \widetilde{\lambda}_{1}^{\mathbf{H}_{2, n, \mathrm{I}}}+1}\right\} \\
& \text { s.t } \sum_{n=1}^{N} a_{1, n}+a_{2, n} \leq P_{\mathrm{T}}
\end{aligned}
$$

where $\widetilde{\lambda}_{1}^{\mathbf{H}_{1, n, \mathrm{I}}}=\frac{\lambda_{1}^{\mathbf{H}_{1, n, \mathrm{I}}}}{\sigma_{1, n}^{2}}$ and $\widetilde{\lambda}_{1}^{\mathbf{H}_{2, n, \mathrm{I}}}=\frac{\lambda_{1}^{\mathbf{H}_{2, n, \mathrm{I}}}}{\sigma_{2, n}^{2}}$. The optimization problem $\overline{\mathcal{P}}_{2}$ is not convex due to the fact that its objective is a sum of quasiconcave functions where each function $f_{n}\left(a_{1, n}, a_{2, n}\right)=\ln _{2}\left\{\frac{a_{1, n} a_{2, n} \widetilde{\lambda}_{1}^{\mathbf{H}_{1, n, \mathrm{I}}} \widetilde{\lambda}_{1}^{\mathbf{H}_{2, n, \mathrm{I}}}}{a_{1, n} \widetilde{\lambda}_{1}^{\mathbf{H}} \mathbf{H}_{1, n, \mathrm{I}}+a_{2, n} \widetilde{\lambda}_{1}^{\mathbf{H}_{2, n, \mathrm{I}}+1}}\right\}$ is a quasiconcave (not concave) function of $a_{1, n}$ and $a_{2, n}$. The quasiconcavity can be verified by showing that the ordered determinants of the bordered Hessian matrix [11] of $f_{n}\left(a_{1, n}, a_{2, n}\right)$ alternate in signs. The derivations are shown in Appendix B.

It is difficult to confirm that the objective function of $\overline{\mathcal{P}}_{2}$ remains quasiconcave, although it is a sum of separable quasiconcave functions. This is due to the fact that in general even the sum of the separable quasiconcave functions may not be quasiconcave [12]. Consequently, we cannot confirm that the local maximum of $\overline{\mathcal{P}}_{2}$ is also the global maximum. However, if $a_{1, n} \widetilde{\lambda}_{1}^{\mathbf{H}_{1, n, \mathrm{I}}}+a_{2, n} \widetilde{\lambda}_{1}^{\mathbf{H}_{2, n, \mathrm{I}}}>>1$ holds true, which is usually the case for moderate and high SNR values of the S$\mathrm{R}$ and R-ID links on the $n$th subcarrier (subcarriers with very low SNR can be allocated zero powers), $f_{n}\left(a_{1, n}, a_{2, n}\right)$ can be shown to be a concave function. Since, the sum of concave 
functions is concave, the approximated objective function in $\overline{\mathcal{P}}_{2}$ becomes concave. As such the maximization of concave function with convex constraints is a convex optimization problem. In the rest of the paper, we deal with the following approximated convex objective function for $\overline{\mathcal{P}}_{2}$ :

$$
\tilde{f} \triangleq \frac{R_{\mathrm{c}}}{2 N} \sum_{n=1}^{N} \ln _{2}\left\{1+\frac{a_{1, n} a_{2, n} \widetilde{\lambda}_{1}^{\mathbf{H}_{1, n, \mathrm{I}}} \widetilde{\lambda}_{1}^{\mathbf{H}_{2, n, \mathrm{I}}}}{a_{1, n} \widetilde{\lambda}_{1}^{\mathbf{H}_{1, n, \mathrm{I}}}+a_{2, n} \widetilde{\lambda}_{1}^{\mathbf{H}_{2, n, \mathrm{I}}}}\right\} .
$$

For the convex optimization problem with the objective function $\widetilde{f}$ and linear constraint of $\overline{\mathcal{P}}_{2}$, the Karush-Kuhn-Tucker (KKT) conditions are necessary and sufficient for the global optimality [13]. The Lagrangian for this problem is given by

$$
L\left(a_{1, n}, a_{2, n}, \mu\right)=-\tilde{f}+\mu\left(\sum_{n=1}^{N} a_{1, n}+a_{2, n}-P_{\mathrm{T}}\right)
$$

where $\mu \geq 0$ is the Lagrangian multiplier. Solving KKT conditions for (16), we find that the optimal solutions for $a_{1, n}$ and $a_{2, n}$ are

$$
a_{i, n}=\frac{\sqrt{\widehat{\lambda}_{j, n}}}{\sqrt{\hat{\lambda}_{1, n}}+\sqrt{\widehat{\lambda}_{2, n}}}\left[\frac{c}{\mu}-\frac{\left[\sqrt{\widehat{\lambda}_{1, n}}+\sqrt{\widehat{\lambda}_{2, n}}\right]^{2}}{\widehat{\lambda}_{1, n} \widehat{\lambda}_{2, n}}\right]^{+}
$$

where $j=2$ if $i=1, j=1$ if $i=2, c=\frac{R_{\mathrm{c}}}{2 N \ln 2},[x]^{+}=$ $\max (0, x), \widehat{\lambda}_{1, n} \triangleq \widetilde{\lambda}_{1}^{\mathbf{H}_{1, n, \mathrm{I}}}, \widehat{\lambda}_{2, n} \triangleq \widetilde{\lambda}_{1}^{\mathbf{H}_{2, n, \mathrm{I}}}$ and $\mu$ should be chosen to satisfy the power constraint in $\overline{\mathcal{P}}_{2}$, which can be done using standard parallel Gaussian waterfilling method.

\section{Optimization with both $\mathrm{EH}$ and ID receivers}

We consider the case in which the $\mathrm{EH}$ receiver exists in the vicinity of the two-hop relay system. In this case, our objective is to develop the source and relay precoders for simultaneously maximizing the power and information transfer. For this purpose, we use the rate-energy (R-E) region which consists of all the achievable rate and energy pairs for a given sum power constraint of the source and relay. Let the $\mathrm{R}-\mathrm{E}$ region be defined as

$$
\begin{gathered}
\mathcal{C}_{R-E}\left(P_{\mathrm{T}}\right) \triangleq\left\{(R, P): R \leq \frac{R_{\mathrm{c}}}{2 N} \sum_{n=1}^{N} \ln _{2}\{1+\right. \\
\left.\frac{\gamma_{1, n} \gamma_{2, n}}{\gamma_{1, n}+\gamma_{2, n}+1}\right\} \\
\gamma_{1, n}=\frac{\left\|\mathbf{H}_{1, n, \mathrm{I}} \mathbf{F}_{1, n}\right\|^{2}}{\sigma_{1, n}^{2}}, \gamma_{2, n}=\frac{\left\|\mathbf{H}_{2, n, \mathrm{I}} \mathbf{F}_{2, n}\right\|^{2}}{\sigma_{2, n}^{2}}, \forall n \\
P \leq \sum_{n=1}^{N}\left\|\mathbf{H}_{1, n, \mathrm{E}} \mathbf{F}_{1, n}\right\|^{2}+\left\|\mathbf{H}_{2, n, \mathrm{E}} \mathbf{F}_{2, n}\right\|^{2} \\
\left.\sum_{n=1}^{N}\left\|\mathbf{F}_{1, n}\right\|^{2}+\left\|\mathbf{F}_{2, n}\right\|^{2} \leq P_{\mathrm{T}}\right\}
\end{gathered}
$$

Let $\left(R_{\mathrm{EH}}, P_{\max }\right)$ and $\left(R_{\max }, P_{\mathrm{ID}}\right)$ be the boundary points of this R-E region corresponding to the maximal power and information transfers, respectively. The source and relay precoders for the boundary point $\left(R_{\mathrm{EH}}, P_{\max }\right)$ are given by (10)-(11), which yield maximum power transfer of $P_{\max }=$ $a K\left(\sum_{n=1}^{N}\left\|\mathbf{H}_{1, n, \mathrm{E}} \widehat{\mathbf{F}}_{1, n}\right\|^{2}+\left\|\mathbf{H}_{2, n, \mathrm{E}} \widehat{\mathbf{F}}_{2, n}\right\|^{2}\right)$ to the EH receiver and the information transfer of $R_{\mathrm{EH}}=0$ to the ID receiver. Note that no transfer of information to the ID receiver is obvious in this case, since the solutions (10)(11) mean that when the best subcarrier is selected, either the source or the relay remains turned off. On the other hand, the source and relay precoders for $\left(R_{\max }, P_{\mathrm{ID}}\right)$ are given by (13) together with (17). With these precoders (i.e., $\overline{\mathbf{F}}_{1, n}$ and $\overline{\mathbf{F}}_{2, n}$ ), the information rate of $R_{\max }$ is achieved whereas the power transferred to the $\mathrm{EH}$ receiver becomes $P_{\mathrm{ID}}=a K\left(\sum_{n=1}^{N}\left\|\mathbf{H}_{1, n, \mathrm{E}} \overline{\mathbf{F}}_{1, n}\right\|^{2}+\left\|\mathbf{H}_{2, n, \mathrm{E}} \overline{\mathbf{F}}_{2, n}\right\|^{2}\right)$. It can be easily seen that for $\bar{P} \leq P_{\mathrm{ID}}$, where $\bar{P} \geq 0$, the maximum rate $R_{\max }$ is achievable with the same $\overline{\mathbf{F}}_{1, n}$ and $\overline{\mathbf{F}}_{2, n}$ that achieve the R-E pair $\left(R_{\max }, P_{\mathrm{ID}}\right)$. The remaining boundary of the R-E region can be determined for the interval $P_{\mathrm{ID}}<\bar{P}<$ $P_{\text {max }}$. For this purpose, we consider the following optimization problem, denoted by $\mathcal{P}_{3}$ with $\mathbf{W}_{1, n} \triangleq \mathbf{F}_{1, n} \mathbf{F}_{1, n}^{H} \succeq 0$ and $\mathbf{W}_{2, n} \triangleq \mathbf{F}_{2, n} \mathbf{F}_{2, n}^{H} \succeq 0$ :

$$
\begin{aligned}
& \max _{\left\{\mathbf{W}_{1, n}, \mathbf{W}_{2, n}, \gamma_{1, n}, \gamma_{2, n}, n \in \mathcal{N}\right\}} \sum_{n=1}^{N} \ln _{2}\left(1+\frac{\gamma_{1, n} \gamma_{2, n}}{\gamma_{1, n}+\gamma_{2, n}}\right) \\
& \text { s.t. } \operatorname{tr}\left(\mathbf{H}_{1, n, \mathrm{I}}^{H} \mathbf{H}_{1, n, \mathrm{I}} \mathbf{W}_{1, n}\right) \geq \gamma_{1, n} \sigma_{1, n}^{2}, \forall n \\
& \operatorname{tr}\left(\mathbf{H}_{2, n, \mathrm{I}}^{H} \mathbf{H}_{2, n, \mathrm{I}} \mathbf{W}_{2, n}\right) \geq \gamma_{2, n} \sigma_{2, n}^{2}, \forall n \\
& \sum_{n=1}^{N} \operatorname{tr}\left(\mathbf{H}_{1, n, \mathrm{E}}^{H} \mathbf{H}_{1, n, \mathrm{E}} \mathbf{W}_{1, n}+\mathbf{H}_{2, n, \mathrm{E}}^{H} \mathbf{H}_{2, n, \mathrm{E}} \mathbf{W}_{2, n}\right) \geq \bar{b} \\
& \operatorname{tr}\left(\mathbf{W}_{1, n}+\mathbf{W}_{2, n}\right) \leq P_{\mathrm{T}} \\
& \mathbf{W}_{1, n} \succeq 0, \mathbf{W}_{2, n} \succeq 0, \forall n,
\end{aligned}
$$

where $\bar{b}=\frac{\bar{P}}{a K}$, the constant term is removed $\frac{R_{c}}{2 N}$ from the objective function and the approximation as in the case of (15) is used. This optimization problem is convex since the constraints are convex and the objective is to maximize the concave function. However, due to additional optimization variables $\left\{\mathbf{W}_{1, n}, \mathbf{W}_{2, n}, n \in \mathcal{N}\right\}$, this problem cannot be solved using the same method as in $\overline{\mathcal{P}}_{2}$. To this end, we rewrite $\widetilde{f}_{n}\left(\gamma_{1, n}, \gamma_{2, n}\right) \triangleq \frac{\gamma_{1, n} \gamma_{2, n}}{\gamma_{1, n}+\gamma_{2, n}}$ as

$$
\begin{aligned}
\tilde{f}_{n}\left(\gamma_{1, n}, \gamma_{2, n}\right)= & \frac{1}{2}\left\{\gamma_{1, n}+\gamma_{2, n}-\gamma_{1, n} \widetilde{\gamma}_{n}^{-1} \gamma_{1, n}\right. \\
& \left.-\gamma_{2, n} \widetilde{\gamma}_{n}^{-1} \gamma_{2, n}\right\}
\end{aligned}
$$

where $\widetilde{\gamma}_{n}=\gamma_{1, n}+\gamma_{2, n}$. Introducing slack variables $\left\{\tau_{n}\right\}_{n=1}^{N}$, the optimization problem (19) is expressed as

$$
\begin{aligned}
& \max _{\left\{\tau_{n}, \mathbf{W}_{1, n}, \mathbf{W}_{2, n}, \gamma_{1, n}, \gamma_{2, n}, n \in \mathcal{N}\right\}} \sum_{n=1}^{N} \ln _{2}\left(1+\tau_{n}\right) \text {, s.t. } \\
& 2 \tau_{n} \leq \gamma_{1, n}+\gamma_{2, n}-\gamma_{1, n} \widetilde{\gamma}_{n}^{-1} \gamma_{1, n}-\gamma_{2, n} \widetilde{\gamma}_{n}^{-1} \gamma_{2, n}, \\
& (19 b), 19 c),(19 d),(19 e),(19 f) .
\end{aligned}
$$

Applying Schur-complement theorem [13] twice for the first constraint of (21), we obtain

$$
\left[\begin{array}{cccc}
\widetilde{\gamma}_{n} & 0 & 0 & 0 \\
0 & \widetilde{\gamma}_{n} & 0 & \gamma_{2, n} \\
0 & 0 & \widetilde{\gamma}_{n} & \gamma_{1, n} \\
0 & \gamma_{2, n} & \gamma_{1, n} & \widetilde{\gamma}_{n}-2 \tau_{n}
\end{array}\right] \succeq 0
$$


As a result, we obtain the following semidefinite programming (SDP) formulation for (19)

$$
\begin{aligned}
& \mathcal{P}_{3}: \max _{\left\{\tau_{n}, \gamma_{i, n}, \mathbf{W}_{i, n}\right\}_{i / n=1}^{2 / N}} \sum_{n=1}^{N} \ln _{2}\left(1+\tau_{n}\right) \text { s.t. }(22), \\
& (19 b), 19 c),(19 d),(19 e),(19 f)
\end{aligned}
$$

which can be efficiently solved using the CVX software [14]. By solving $\mathcal{P}_{3}$ for $P_{\mathrm{ID}}<\bar{P}<P_{\max }$, we obtain the optimal rate solutions that form the boundary of the R-E region over the interval $\left(R_{\mathrm{EH}}=0\right)<R<R_{\max }$. Note that, in our case, the optimal $\left\{\mathbf{F}_{i, n}\right\}_{i / n=1}^{2 / N}$ can be recovered from the optimal $\left\{\mathbf{W}_{i, n}\right\}_{i / n=1}^{2 / N}$ without any loss of optimality.

\section{NUMERICAL RESULTS}

We plot the boundary of the R-E region using numerical results. The results are obtained by averaging over 100 independent realizations of frequency selective MIMO channels, that are generated using MATLAB's mimochan function. We take $5 \mathrm{MHz}$ bandwidth, carrier frequency of $2.6 \mathrm{GHz}, N=16$ and consider that channels have root mean-square (rms) delays up to $45 \mathrm{~ns}$ and maximum delay spread up to $410 \mathrm{~ns}$. In particular, we simulate channels with the following power delay profiles (PDP) [15]

$$
\begin{array}{ll}
\mathrm{PDP}_{1}: & \boldsymbol{\tau}^{1}=[0,50,110,170,290,310] \mathrm{ns} \\
& \mathbf{p}^{1}=[0,-3,-10,-18,-26,-32] \mathrm{dB}, \text { and } \\
\mathrm{PDP}_{2}: & \boldsymbol{\tau}^{2}=[0,110,190,410] \mathrm{ns}, \\
& \mathbf{p}^{2}=[0,-9.7,-19.2,-22.8] \mathrm{dB}
\end{array}
$$

where $\tau^{i}$ and $\mathbf{p}^{i},(i=1,2)$, represent relative path delays and corresponding average powers, respectively. For all results, we take $\sigma_{1, n}^{2}=\sigma_{2, n}^{2}=\sigma^{2} / N$, and $n_{\mathrm{s}}=n_{\mathrm{r}}=2$, i.e., both the source and relay use the Alamouti code [7] $\left(R_{\mathrm{c}}=1\right)$. The average SNR is defined as $\gamma_{\mathrm{av}} \triangleq \widetilde{P}_{\mathrm{T}} / \sigma^{2}$. We vary $\widetilde{P}_{\mathrm{T}}$ with $\sigma^{2}=1$ and take $n_{\mathrm{d}}=n_{\mathrm{e}}=2$. Figure 2 plots the

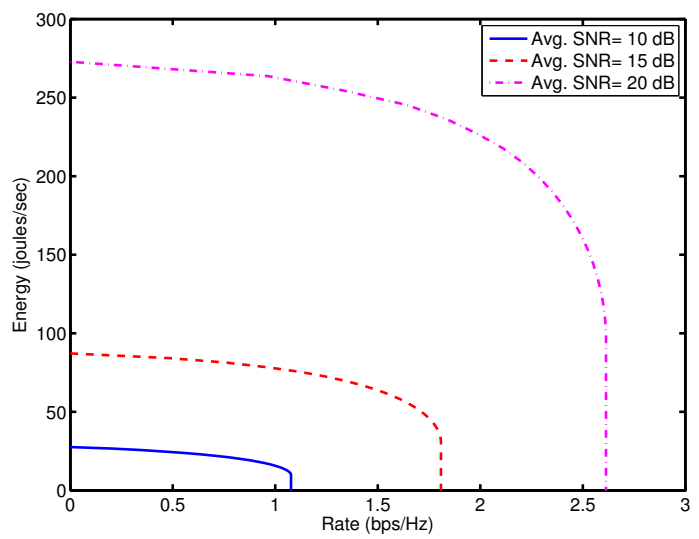

Fig. 2. R-E region tradeoff for the channels with $\mathrm{PDP}_{1}$.

tradeoff between the maximum energy harvested by the $\mathrm{EH}$ receiver and the maximum information rate transferred to the ID receiver for different values of $\gamma_{\text {av }}$ for a channel model with $\mathrm{PDP}_{1}$. The rms delay for this channel model is $35 \mathrm{~ns}$ whereas a flat Doppler spectrum is used. It can be observed from this figure that energy transfer to the $\mathrm{EH}$ receiver increases with almost the same factor as that of increment in $\gamma_{\mathrm{av}}$, whereas this is not the case for information transfer to the ID receiver. This can be understood from the fact that energy, or roughly power, is linearly proportional to SNR whereas rate is only proportional to logarithmic of SNR. In Fig. 3, we display the boundary of the R-E region for different $\gamma_{\mathrm{av}}$ and doppler frequencies $\left(f_{D_{1}}, f_{D_{2}}\right)$ using the channel model with $\mathrm{PDP}_{2}$. The rms delay for this channel is $45 \mathrm{~ns}$ and we take $f_{D_{1}}=4.6$ $\mathrm{Hz}$ and $f_{D_{2}}=104.2 \mathrm{~Hz}$ corresponding to the speeds of 2 $\mathrm{Km} / \mathrm{hr}$ and $45 \mathrm{Km} / \mathrm{hr}$, respectively. This figure also shows that both energy and information transfer increase as $\gamma_{\mathrm{av}}$ increases. However, energy increases in a similar proportion as the increment in $\gamma_{\text {av }}$, whereas the rate not. As expected, the boundary of R-E shrinks as doppler spread increases.

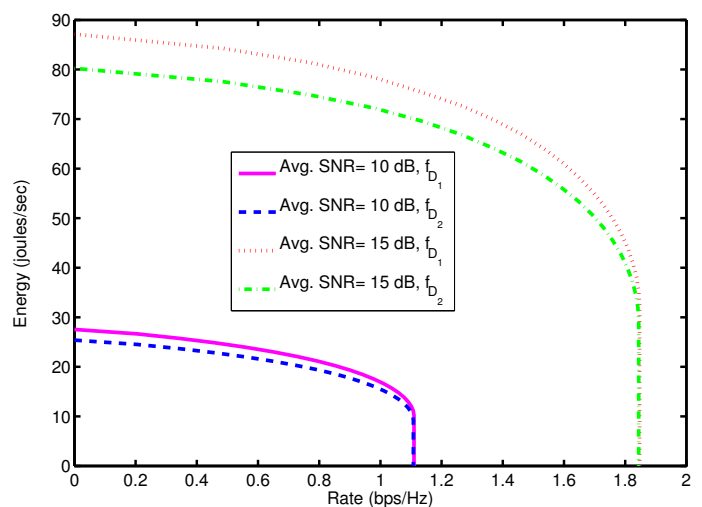

Fig. 3. R-E region tradeoff for the channels with $\mathrm{PDP}_{2}$.

\section{Conclusions}

We have provided the performance limits of the OSTBC based MIMO-OFDM relay system that allows low-powered wireless devices in its vicinity to harvest energy. The tradeoffs in information rate and energy transfer were demonstrated from the boundary of the R-E region which is obtained by solving joint source and relay precoder optimization problems.

\section{APPENDIX A}

Let the EDs for $\mathbf{F}_{1, n} \mathbf{F}_{1, n}^{H}$ and $\mathbf{F}_{2, n} \mathbf{F}_{2, n}^{H}$ be given by $\mathbf{F}_{1, n} \mathbf{F}_{1, n}^{H} \triangleq \mathbf{U}_{\mathbf{F}_{1, n}} \boldsymbol{\Sigma}_{\mathbf{F}_{1, n}} \mathbf{U}_{\mathbf{F}_{1, n}}^{H}$ and $\mathbf{F}_{2, n} \mathbf{F}_{2, n}^{H} \triangleq$ $\mathbf{U}_{\mathbf{F}_{2, n}} \boldsymbol{\Sigma}_{\mathbf{F}_{2, n}} \mathbf{U}_{\mathbf{F}_{2, n}}^{H}$, respectively, where $\mathbf{U}_{\mathbf{F}_{1, n}} \mathbf{U}_{\mathbf{F}_{1, n}}^{H}=\mathbf{I}_{n_{\mathrm{s}}}$, $\mathbf{U}_{\mathbf{F}_{2, n}} \mathbf{U}_{\mathbf{F}_{2, n}}^{H}=\mathbf{I}_{n_{\mathrm{r}}}$. Without loss of generality (w.1.o.g), the diagonal entries $\left\{\sigma_{j}^{\mathbf{F}_{1, n}}\right\}_{j=1}^{n_{\mathrm{s}}}$ and $\left\{\sigma_{k}^{\mathbf{F}_{2, n}}\right\}_{k=1}^{n_{\mathrm{r}}}$ of $\boldsymbol{\Sigma}_{\mathbf{F}_{1, n}}$ and $\boldsymbol{\Sigma}_{\mathbf{F}_{2, n}}$ are assumed to be in the decreasing order. Then, we can express $s_{i, n} \triangleq \operatorname{tr}\left(\mathbf{H}_{i, n, \mathrm{E}} \mathbf{F}_{i, n} \mathbf{F}_{i, n}^{H} \mathbf{H}_{i, n, \mathrm{E}}^{H}\right)$ as

$$
\begin{aligned}
s_{i, n} & =\operatorname{tr}\left(\mathbf{U}_{\mathbf{F}_{i, n}}^{H} \mathbf{U}_{\mathbf{H}_{i, n, \mathrm{E}}} \boldsymbol{\Lambda}_{\mathbf{H}_{i, n, \mathrm{E}}} \mathbf{U}_{\mathbf{H}_{i, n, \mathrm{E}}}^{H} \mathbf{U}_{\mathbf{F}_{i, n}} \boldsymbol{\Sigma}_{\mathbf{F}_{i, n}}\right) \\
& \leq \operatorname{tr}\left(\boldsymbol{\Lambda}_{\mathbf{H}_{i, n, \mathrm{E}}} \boldsymbol{\Sigma}_{\mathbf{F}_{i, n}}\right)
\end{aligned}
$$

where the equality holds only if $\mathbf{U}_{\mathbf{F}_{i, n}}=\mathbf{U}_{\mathbf{H}_{i, n, \mathrm{E}}}$. Substituting this result, $\mathcal{P}_{1}$ can be expressed as

$$
\begin{aligned}
& \max _{\substack{\mathbf{F}_{j} \\
\mathbf{m}_{1, n}, \mathbf{F}_{2, n}}} \sum_{n=1}^{N}\left\{\sum_{j=1}^{r_{1}} \lambda_{j}^{\mathbf{H}_{1, n, \mathrm{E}}} \sigma_{j}^{\mathbf{F}_{1, n}}+\sum_{k=1}^{r_{2}} \lambda_{k}^{\mathbf{H}_{2, n, \mathrm{E}}} \sigma_{k}^{\mathbf{F}_{2, n}}\right\} \\
& \text { s.t. } \sum_{n=1}^{N}\left[\sum_{j=1}^{r_{1}} \sigma_{j}^{\mathbf{F}_{1, n}}+\sum_{k=1}^{r_{2}} \sigma_{k}^{\mathbf{F}_{2, n}}\right] \leq P_{\mathrm{T}},
\end{aligned}
$$


which, can be equivalently expressed as

$$
\begin{aligned}
& \max _{\sigma_{1}^{\mathbf{F}_{1, n}}, \sigma_{1}^{\mathbf{F}_{2, n}}} \sum_{n=1}^{N}\left\{\lambda_{1}^{\mathbf{H}_{1, n, \mathrm{E}}} \sigma_{1}^{\mathbf{F}_{1, n}}+\lambda_{k}^{\mathbf{H}_{2, n, \mathrm{E}}} \sigma_{1}^{\mathbf{F}_{2, n}}\right\} \\
& \text { s.t. } \sum_{n=1}^{N} \sigma_{1}^{\mathbf{F}_{1, n}}+\sigma_{1}^{\mathbf{F}_{2, n}} \leq P_{\mathrm{T}},
\end{aligned}
$$

with $\left\{\sigma_{j}^{\mathbf{F}_{1, n}}\right\}_{j \neq 1}^{n_{\mathrm{s}}}=0$ and $\left\{\sigma_{k}^{\mathbf{F}_{1, n}}\right\}_{k \neq 1}^{n_{\mathrm{r}}}=0$. The optimization problem (25) is a linear programming problem, for which the optimal solutions are readily given by, either

$\sigma_{1}^{\mathbf{F}_{1, n}}=\left\{\begin{array}{c}P_{\mathrm{T}}, \text { if } \lambda_{1}^{\mathbf{H}_{1, n, \mathrm{E}}}>\left\{\lambda_{1}^{\mathbf{H}_{1, \tilde{n}, \mathrm{E}}}, \lambda_{1}^{\mathbf{H}_{2, \bar{n}, \mathrm{E}}}\right\}_{\widetilde{n} \neq n, \widetilde{n}, \bar{n}=1}^{N}, \\ 0 \text { otherwise }\end{array}\right.$

in which $\left\{\sigma_{1}^{\mathbf{F}_{2, n}}\right\}_{n=1}^{N}=0$, or

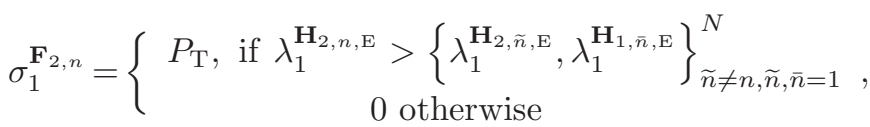

in which $\left\{\sigma_{1}^{\mathbf{F}_{1, n}}\right\}_{n=1}^{N}=0$. Using these solutions for $\sigma_{1}^{\mathbf{F}_{1, n}}$ and $\sigma_{1}^{\mathbf{F}_{2, n}}$, we obtain (11).

\section{APPENDIX B}

For notational convenience, we define $\lambda_{1 n} \triangleq \widetilde{\lambda}_{1}^{\mathbf{H}_{1, n, \mathrm{I}}}$, $\lambda_{2 n} \triangleq \widetilde{\lambda}_{1}^{\mathbf{H}_{2, n, \mathrm{I}}}, t_{1 n} \triangleq a_{1, n}$, and $t_{2 n} \triangleq a_{2, n}$. Then, the resulting function $f_{n}\left(a_{1, n}, a_{2, n}\right)$ can be expressed as $f_{n}\left(a_{1, n}, a_{2, n}\right) \triangleq \frac{1}{\ln 2} f_{n}\left(t_{1 n}, t_{2 n}\right)$ with $f_{n}\left(t_{1 n}, t_{2 n}\right) \triangleq$ $\ln \left(1+\frac{t_{1 n} \lambda_{1 n} t_{2 n} \lambda_{2 n}}{t_{1 n} \lambda_{1 n}+t_{2 n} \lambda_{2 n}+1}\right)$. The bordered Hessian matrix [11] for $f_{n}\left(t_{1 n}, t_{2 n}\right)$ is defined as

$$
\mathbf{H f}_{n}=\left[\begin{array}{ccc}
0 & \frac{\delta f_{n}}{\delta t_{1 n}} & \frac{\delta f_{n}}{\delta t_{2 n}} \\
\frac{\delta f_{n}}{\delta t_{1 n}} & \frac{\delta^{2} f_{n}}{\delta t_{1 n}^{2}} & \frac{\delta^{2} f_{n}}{\delta t_{1 n} \delta t_{2 n}} \\
\frac{\delta f_{n}}{\delta t_{2 n}} & \frac{\delta^{2} f_{n}}{\delta t_{2 n} \delta t_{1 n}} & \frac{\delta^{2} f_{n}}{\delta t_{2 n}^{2}}
\end{array}\right]
$$

The first-order partial derivatives of $f_{n}$ with respect to $t_{1 n}$ and $t_{2 n}$ are given by

$$
\begin{aligned}
\frac{\delta f_{n}}{\delta t_{1 n}} & =\frac{\lambda_{1 n} \lambda_{2 n} t_{2 n}}{\left(1+\lambda_{1 n} t_{1 n}\right)\left(1+\lambda_{1 n} t_{1 n}+\lambda_{2 n} t_{2 n}\right)} \\
\frac{\delta f_{n}}{\delta t_{2 n}} & =\frac{\lambda_{1 n} \lambda_{2 n} t_{1 n}}{\left(1+\lambda_{2 n} t_{2 n}\right)\left(1+\lambda_{1 n} t_{1 n}+\lambda_{2 n} t_{2 n}\right)}
\end{aligned}
$$

which shows that the function $f_{n}\left(t_{1 n}, t_{2 n}\right)$ increases monotonically in $t_{1 n}$ and $t_{2 n}$. The corresponding second order partial derivatives are then given by

$$
\begin{aligned}
\frac{\delta^{2} f_{n}}{\delta t_{1 n}^{2}} & =\frac{-\lambda_{1 n}^{2} \lambda_{2 n} t_{2 n}\left(2+2 \lambda_{1 n} t_{1 n}+\lambda_{2 n} t_{2 n}\right)}{\left(1+\lambda_{1 n} t_{1 n}\right)^{2}\left(1+\lambda_{1 n} t_{1 n}+\lambda_{2 n} t_{2 n}\right)^{2}} \\
\frac{\delta^{2} f_{n}}{\delta t_{2 n}^{2}} & =\frac{-\lambda_{2 n}^{2} \lambda_{1 n} t_{1 n}\left(2+2 \lambda_{2 n} t_{2 n}+\lambda_{1 n} t_{1 n}\right)}{\left(1+\lambda_{2 n} t_{2 n}\right)^{2}\left(1+\lambda_{1 n} t_{1 n}+\lambda_{2 n} t_{2 n}\right)^{2}}
\end{aligned}
$$

which shows that $\frac{\delta^{2} f_{n}}{\delta t_{1 n}^{2}}$ and $\frac{\delta^{2} f_{n}}{\delta t_{2 n}^{2}}$ are negative for all possible $t_{1 n}, t_{2 n}$. Similarly, we obtain

$$
\begin{aligned}
\frac{\delta^{2} f_{n}}{\delta t_{1 n} \delta t_{2 n}} & =\frac{\lambda_{1 n} \lambda_{2 n}}{\left(1+\lambda_{1 n} t_{1 n}+\lambda_{2 n} t_{2 n}\right)^{2}} \\
\frac{\delta^{2} f_{n}}{\delta t_{2 n} \delta t_{1 n}} & =\frac{\delta^{2} f_{n}}{\delta t_{1 n} \delta t_{2 n}} .
\end{aligned}
$$

Let $D_{n}^{i}$ denote the $i$ th-ordered determinant of the bordered Hessian matrix $\mathbf{H f}_{n}$. Then, we have

$$
\begin{aligned}
D_{n}^{1}= & -\left[\frac{\delta f_{n}}{\delta t_{1 n}}\right]^{2} \\
D_{n}^{2}= & 2 \frac{\delta f_{n}}{\delta t_{1 n}} \frac{\delta f_{n}}{\delta t_{2 n}} \frac{\delta^{2} f_{n}}{\delta t_{1 n} \delta t_{2 n}}-\left[\frac{\delta f_{n}}{\delta t_{1 n}}\right]^{2} \frac{\delta^{2} f_{n}}{\delta t_{2 n}^{2}} \\
& -\left[\frac{\delta f_{n}}{\delta t_{2 n}}\right]^{2} \frac{\delta^{2} f_{n}}{\delta t_{1 n}^{2}} .
\end{aligned}
$$

Note that $D_{n}^{1} \leq 0$ since $\frac{\delta f_{n}}{\delta t_{1 n}} \geq 0$, whereas $D_{n}^{2} \geq 0$ since $\frac{\delta f_{n}}{\delta t_{1 n}} \geq 0, \frac{\delta f_{n}}{\delta t_{2 n}} \geq 0, \frac{\delta^{2} f_{n}}{\delta t_{1 n}^{2}} \leq 0, \frac{\delta^{2} f_{n}}{\delta t_{2 n}^{2}} \leq 0$ and $\frac{\delta^{2} f_{n}}{\delta t_{1 n} \delta t_{2 n}} \geq 0$. The facts that $D_{n}^{1} \leq 0$ and $D_{n}^{2} \geq 0$ verify that $f_{n}\left(t_{1 n}, t_{2 n}\right)$ is a quasiconcave function [11]. Although $\frac{\delta^{2} f_{n}}{\delta t_{1 n}^{2}} \leq 0, \frac{\delta^{2} f_{n}}{\delta t_{2 n}^{2}} \leq 0$, this function is not concave since $\frac{\delta^{2} f_{n}}{\delta t_{1 n}^{2}} \frac{\delta^{2} f_{n}}{\delta t_{2 n}^{2}}-\left[\frac{\delta^{2} f_{n}}{\delta t_{1 n} \delta t_{2 n}}\right]^{2} \geq 0$ does not hold for all $t_{1 n}$ and $t_{2 n}$. The concavity holds only under some conditions, e.g., for all $t_{1 n}, t_{2 n}$, where $\frac{t_{1 n} \lambda_{1 n} t_{2 n} \lambda_{2 n}}{t_{1 n} \lambda_{1 n}+t_{2 n} \lambda_{2 n}+1} \geq 1 / 3$ for given $\lambda_{1 n}$ and $\lambda_{2 n}$. This is usually the case under high SNR assumption.

\section{REFERENCES}

[1] R. Want, "Enabling ubiquitous sensing with RFID," IEEE Computer, vol. 37, pp. 84-86, Apr. 2004.

[2] F. Zhang et al., "Wireless energy transfer platform for medical sensors and implantable devices," in Proc. IEEE EMBS 31st Annual Int. Conf., pp. 1045-1048, Sept. 2009.

[3] R. Zhang and C. K. Ho, "MIMO broadcasting for simultaneous wireless information and power transfer," in Proc. IEEE Globecom'11, pp. 1-5, Dec. 2011.

[4] L. R. Varshney, "Transporting information and energy simultaneously," in Proc. IEEE Int. Symp. Inf. Theory (ISIT), pp. 16121616, July 2008.

[5] P. Grover and A. Sahai, "Shannon meets Tesla: wireless information and power transfer," in Proc. IEEE Int. Symp. Inf. Theory (ISIT), pp. 2363-2367, June 2010.

[6] B. K. Chalise, Y. D. Zhang, and M. G. Amin, "Energy harvesting in an OSTBC based amplify-and-forward MIMO relay system," in Proc. IEEE ICASSP, Mar. 2012, Kyoto, Japan.

[7] V. Tarokh, H. Jafharkani, and A. R. Calderbank, "Space-time block codes from orthogonal designs," IEEE Trans. Info. Theo., vol. 45, no. 5, pp. 1456-1467, July 1999.

[8] I. Hammerstrom and A. Wittneben, "Power allocation schemes for amplify-and-forward MIMO-OFDM relay links," IEEE Trans. Wireless Commun., vol. 6, no. 8, Aug. 2007.

[9] B. K. Chalise and L. Vandendorpe, "MIMO relay design for multipoint-to-multipoint communications with imperfect channel state information," IEEE Trans. Sig. Proc., vol. 57, no. 7, pp. 2785-2796, July 2009.

[10] A. Hjorugnes and D. Gesbert, "Precoding of orthogonal-space time block codes in arbitrarily correlated MIMO channels: Iterative and closed-form solutions," IEEE Trans. Wireless Commun., vol. 6, no. 3, pp. 1072-1082, Mar. 2007.

[11] K. J. Arrow and A. C. Enthoven, "Quasi-concave programming," Econometrica, Oct. 1961.

[12] Y. Almogy and 0. Levin, "A class of fractional programming," Operations Research, vol. 19, no. 1, pp. 57-67, 1971.

[13] S. Boyd and L. Vandenberghe, Convex Optimization, Cambridge University Press, 2004.

[14] M. Grant and S. Boyd, CVX: Matlab Software for Disciplined Convex Programming, http://stanford.edu/־boyd/cvx.

[15] ITU-R Recommendation M.1225, "Guidelines for evaluation of radio transmission technologies for IMT-2000," 1997. 